Research Paper

\title{
MicroRNA-320 targeting neuropilin 1 inhibits proliferation and migration of vascular smooth muscle cells and neointimal formation
}

\author{
Hongqiang Li, Jinlong Zhao, Baoxin Liu, Jiachen Luo, Zhiqiang Li, Xiaoming Qin, Yidong Wei ${ }^{\bowtie}$ \\ Department of Cardiology, Shanghai Tenth People's Hospital, Tongji University School of Medicine, 301 Middle Yanchang Road, Jingan District, Shanghai, \\ People's Republic of China \\ $\triangle$ Corresponding author: Yidong Wei MD, Ph.D. Department of Cardiology, Shanghai Tenth People's Hospital, Tongji University School of Medicine. 301 \\ Middle Yanchang Road, Jingan District, Shanghai, People's Republic of China. Telephone number: +86-18917683409 Fax number: 021-66301771 E-mail address: \\ ywei@tongji.edu.cn. (Yidong Wei). \\ (c) Ivyspring International Publisher. This is an open access article distributed under the terms of the Creative Commons Attribution (CC BY-NC) license \\ (https:// creativecommons.org/licenses/by-nc/4.0/). See http://ivyspring.com/terms for full terms and conditions.
}

Received: 2018.06.25; Accepted: 2018.10.18; Published: 2019.01.01

\begin{abstract}
This study shows that microRNA-320 (miR-320) is associated with many important cell functions, including cell differentiation, proliferation, migration, and apoptosis. However, the role of miR-320 in vascular smooth muscle cells (VSMCs) and proliferative vascular diseases is still completely unclear. In our study, we found that the expression of miR-320 in human VSMCs after PDGF stimulation was significantly down-regulated in time- and dose-dependent manner. Function analyses identified that miR-320 could inhibit the proliferation and migration of VSMCs in both basal and PDGF-stimulated conditions. Furthermore, Neuropilin 1 (NRPI) was demonstrated as a direct target of miR-320 in Luciferase reporter assays and miR-320 overexpression inhibited the expression of NRPI with or without PDGF treatment. Finally, miR-320 was markedly decreased in mice carotid arteries after ligated injury, while the restoration of miR-320 via Ad-miR-320 attenuated neointimal hyperplasia by declining the NRPI expression. The results confirmed that miR-320 regulated proliferation and migration of VSMCs and neointimal formation by targeting NRPI. These novel findings implied that the regulation of NRPI expression by miR-320 has important significance in the early diagnosis and treatment of proliferation vascular diseases.
\end{abstract}

Key words: miR-320; proliferation; migration; vascular smooth muscle cell; neointimal formation

\section{Introduction}

Vascular smooth muscle cells (VSMCs) are located in the middle layer of the vascular wall. Abnormal proliferation and migration of VSMCs induced by local stimulus play an important role in the development of cardiovascular diseases, such as restenosis after coronary artery bypass and stent, atherosclerosis, hypertension, and pulmonary artery hypertension [1, 2]. Unlike terminally differentiated myocardium and nerve cell, VSMCs could alternate phenotype from the differentiated contractile phenotype to dedifferentiated synthetic phenotype stimulated by environmental factors. In physiological conditions, VSMCs has been shown not to proliferate and migrate. However, a significant increase in proliferation and migration could be regulated by injury and growth factors, especially the platelet-derived growth factor (PDGF). PDGF, which is derived from platelets and vascular cells following injury, has been demonstrated as one of the most important cytokines to promote the proliferation and migration of smooth muscle cells. Furthermore, pharmacological and genetic approaches have shown a causal relationship between PDGF signaling and cardiovascular diseases[3].

MicroRNAs (miRNAs) are a class of highly conserved, noncoding, single-stranded RNA of $\approx 22$ nucleotides, which perform pivot biological functions via promotion of degradation and translation 
inhibition of relative target mRNAs [4,5]. Increasing studies have found that miRNAs are associated with almost all important cell functions, including cell differentiation, proliferation, migration, and apoptosis [6-9]. Meanwhile, miRNAs also play an important role in the regulation of cardiovascular physiological development and cardiovascular diseases [10, 11]. In fact, many miRNAs, including miR-1, miR-21， miR-24， miR-145， miR-221/222, miR-663 were testified to modulate phenotypic switch of VSMCs and neointima formation after vascular injury [12-17]. In response to vascular injury and PDGF stimulation, the expression of miR-1, miR-21, miR-24, miR-221/222 was increased, which resulted in accelerating proliferation and migration of VSMCs. However, miR-145 was one of the highest expressed miRNAs in vascular walls, which was sharply down-regulated in rat models of carotid balloon injury. Furthermore, the restoration of decreased miR-145 could attenuate intimal hyperplasia [15]. miR-320 is closely related to the proliferation, migration, and apoptosis of many kinds of cancer cells, such as oral squamous cell carcinoma cell lines, cholangiocarcinoma cells, prostate cancer cells, colorectal cancer cells [18-21]. However, the effects of miR-320 on VSMCs and proliferative vascular diseases are still completely unclear.

In the current study, we demonstrated that miR-320 inhibited the proliferation and migration of human VSMCs by targeting Neuropilin 1 (NRP1) in vitro. Moreover, we found that miR-320 was dramatically decreased in ligated mouse carotid injury and restoration of miR-320 attenuate intimal hyperplasia in vivo, suggesting that miR-320 could be used as a new phenotypic marker and therapeutic target.

\section{Materials and methods}

\section{Cell culture and transfection}

HEK293T cells were cultured in Dulbecco's modified Eagle's medium (DMEM) with 10\% fetal bovine serum (FBS) and 1\% penicillin-streptomycin. Human aortic smooth cells were obtained from ScienCell Research Laboratories (USA, Cat. No.6110) and maintained in Smooth Muscle Cell Medium (SMCM, Cat. No.1101), containing 2\%FBS and 1\% penicillin-streptomycin. Both cells were incubated in humid conditions at $37^{\circ} \mathrm{C}$ at $5 \% \mathrm{CO} 2$. The cells from 3 passages to 5 passages were used in the experiment. Human PDGF-BB was purchased from R\&D Systems. VSMCs were starved for 24 hours with no serum and stimulated with PDGF-BB (20ng/ml). miR-320 mimic (Cat. No. miR20000903) and inhibitor (Cat. No. miR10000903), si-NRP1 (Cat. No. StB0002363) and corresponding negative control were obtained from Ribobio (Guangzhou, China). The final transfection concentrations of miR-320 mimic and inhibitor, si-NRP1 were $50 \mathrm{nM}, 100 \mathrm{nM}$, and $50 \mathrm{nM}$, respectively, using lipofectamine 2000 (Invitrogen, USA) by the manufacturer's instructions.

\section{Quantitative real-time PCR}

Total RNA from human VSMCs and mice carotid artery tissues was extracted using Trizol reagent (Invitrogen, USA). cDNA was synthesized by the Prime Script RT Master Mix(Takara, Japan) and detected by SYBR Premix EX TaqII(Takara, Japan) by Light Cycler 96 (BIOTECON Diagnostics, Roche, Switzerland). The primer sequences used in the experiment were as follows: miR-320(RT primer: 5'-GTC GTA TCC AGT GCG TGT CGT GGA GTC GGC AAT TGC ACT GGA TAC GAC TCG CCC T-3'; forward primer: 5'-AAA AGC TGG GTT GAG AGG GCG A-3'; reverse primer: 5'-CAG TGC GTG TCG TGG AGT-3'). The abundance of miR-320 relative to U6 was quantified by the $2^{-\Delta \Delta \mathrm{Ct}}$ method.

\section{Western blot}

The protein from VSMCs and tissues was isolated by cell lysis buffer (9803s, CST) with protease inhibitor cocktail (Roche, USA) and centrifuged for $15 \mathrm{~min}$ at $12000 \mathrm{rpm}$ at $4^{\circ} \mathrm{C}$. Protein lysates were electrophoresed using SDS-PAGE and transferred to PVDF membranes. The membranes were blocked with 5\% non-fat milk powder, incubated with primary antibody against PCNA (13110s, 1:1000, rabbit, CST), SMA(ab32575,1:1000, rabbit, Abcam), NRP1 (ab81321, 1:1000, rabbit, Abcam), GFP(GTX113617, 1:3000, rabbit,GeneTex), $\beta$-tubulin (2128s, 1:5000, rabbit, CST) overnight at $4^{\circ} \mathrm{C}$, followed by binding to horseradish peroxidase-conjugated secondary antibody. The bands were scanned using Amersham Imager 600 ECL system (GE Healthcare, USA).

\section{Cell proliferation and wound-healing assay}

Cells were cultured in a 12-well plate, transfected for $48 \mathrm{~h}$, starved for $12 \mathrm{~h}$, stimulated with or without PDGF-BB $(20 \mathrm{ng} / \mathrm{ml})$ for $24 \mathrm{~h}$. EdU was added for the last $4 \mathrm{~h}$ of stimulation. Cell proliferation was evaluated based on EdU incorporation, using Click-iT EdU Imaging Kit (Invitrogen, USA) according to manufacturer's protocol. The stained cells were photographed using an Olympus IX83 microscope (OLYMPUS, Japan) and calculated the percentage of positive proliferating cells.

Transfected cells were starved in serum-free medium for $24 \mathrm{~h}$. A scratch was made in the center of the $6 \mathrm{~cm}$ dish using 200ul tips and treated with PDGF-BB $(20 \mathrm{ng} / \mathrm{ml})$ for $24 \mathrm{~h}$. Images of cell migration 
before and after stimulation were taken and the migration areas were calculated.

\section{Luciferase assay}

HEK293T cells were cultured in 24-well plate, co-transfected with luciferase reporter plasmid and miR-320 mimic or NC mimic. 48h after transfection, luciferase activity was detected by Dual-Luciferase Reporter Assay System (Promega).

\section{Mouse carotid artery ligation and adenovirus transfection}

Male C57BL/ 6 mice (20-25 g) were anesthetized with an intraperitoneal injection of $3.5 \%$ chloral hydrate $(0.1 \mathrm{ml} / 10 \mathrm{~g})$. The left common carotid artery was ligated with 6-0 silk suture proximal to the bifurcation and the blood flow was completely blocked. As for the control group, the right common carotid artery was subjected to the same procedure except for ligation [22]. The common carotid artery was fully dissociated from surrounding tissues. Ad-miR-320 (Cat. No. H8709) or Ad-GFP (Cat. No. H201) was purchased from OBIO Technology (Shanghai, China), dissolved in Pluronic F127 gel (20\% wt/vol, Sigma, USA) and placed around the blood vessel. At 7,14 or $28 \mathrm{~d}$ after surgery, carotid artery tissues were harvested after systemic perfusion with PBS, fixed with $4 \%$ paraformaldehyde and embedded in paraffin. The tissue sections $(5 \mu \mathrm{m})$ were stained with hematoxylin and eosin for histological and morphometric analysis. All the animal experiments were approved by the Ethics Committee of Shanghai Tenth People's Hospital.

\section{Immunofluorescence}

After antigen retrieval with sodium citrate buffer (PH 6.0), the tissues sections were blocked with $1 \%$
BSA, incubated with primary antibodies to PCNA(2586T, 1:100, mouse, CST), SMA(ab32575,1:100, rabbit, Abcam; sc-53142,1:100, mouse, SCBT), NRP1(ab81321, 1:100, rabbit, Abcam) overnight at $4^{\circ} \mathrm{C}$. From this step forward, samples should be protected from light and incubated using secondary antibody (1:200, YEPSEN) for $60 \mathrm{~min}$ at room temperature. The nuclei were stained with DAPI.

\section{Statistical analysis}

The data were represented as mean \pm SD and analyzed with SPSS software (version 20.0). The differences between two groups were determined by independent sample t-test. The comparisons between multiple groups were performed using one-way analysis of variance. $\mathrm{P}<0.05$ was considered statistically significant.

\section{Results}

\section{MiR-320 expression is down-regulated in proliferative VSMCs}

This was demonstrated by this study that miR-320, miR-218, and miR-194 were highly expressed in normal rat carotid artery [23]. To confirm these results, human aortic VSMCs were stimulated by PDGF-BB and these miRNAs levels were evaluated by quantitative reverse-transcription PCR (qRT-PCR). We found that the expression of miR-218 and miR-194 did not change after treatment with PDGF-BB (Figure 1A). In contrast, PDGF-BB resulted in dramatic down-regulation of miR-320 expression by qRT-PCR, displaying time and dose dependence (Figure $1 \mathrm{~B}$ and 1C).
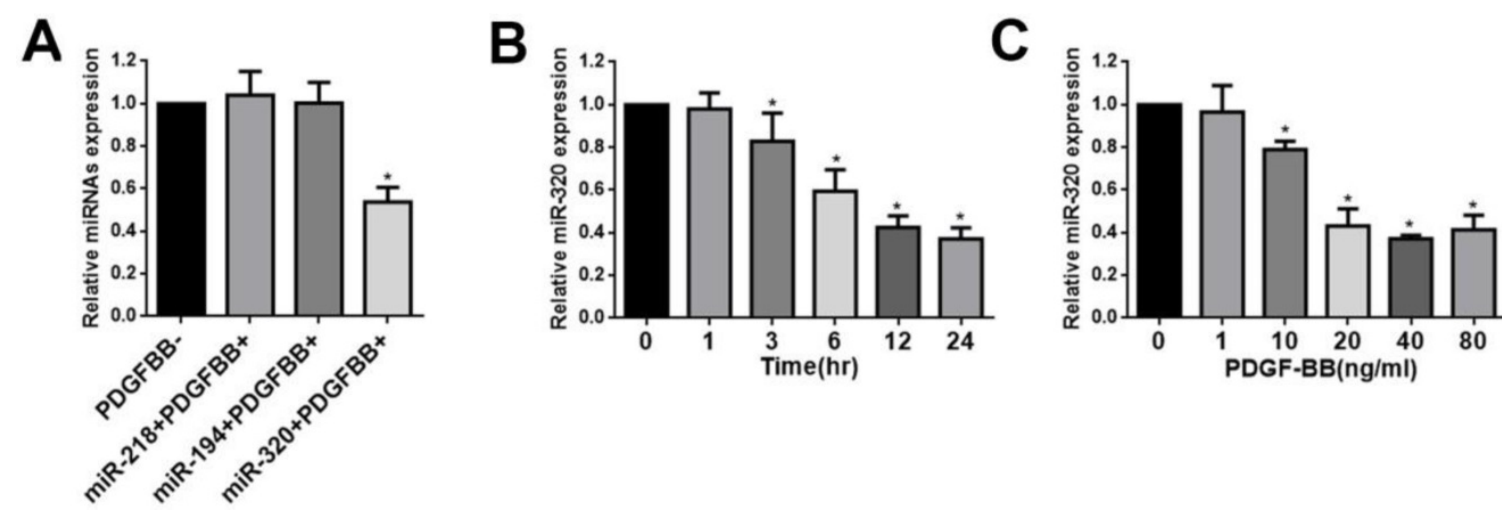

Figure 1. miR-320 is down-regulated in VSMCs by PDGF. (A) VSMCs were starved for 24h in no-serum condition and treated with PDGF-BB (20ng/ml) for 24h. MiR-320 expression was decreased rather than miR-218 and miR-194 by qRT-PCR. (B) MiR-320 expression displayed a time-dependent decrease in VSMCs treated with PDGF-BB (20ng/ml) by qRT-PCR. (C) MiR-320 expression displayed a dose-dependent decrease in VSMCs treated with PDGF-BB for $24 \mathrm{~h}$ by qRT-PCR. $\mathrm{n}=3 ; * \mathrm{P}<0.05$ vs that without PDGF-BB treatment. 
A

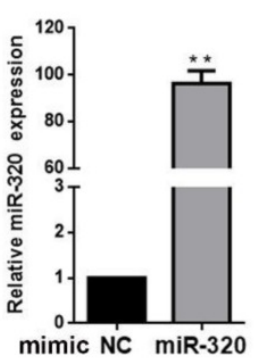

C

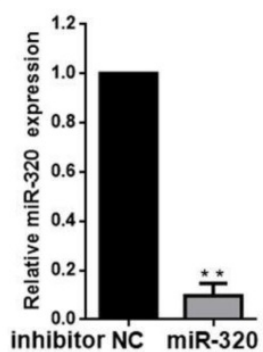

B

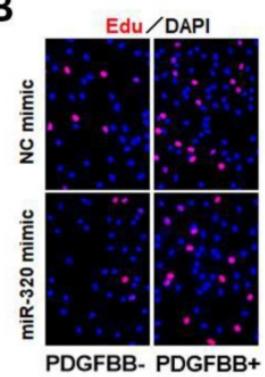

D

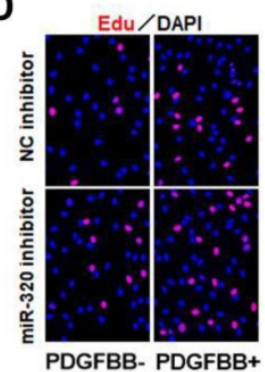

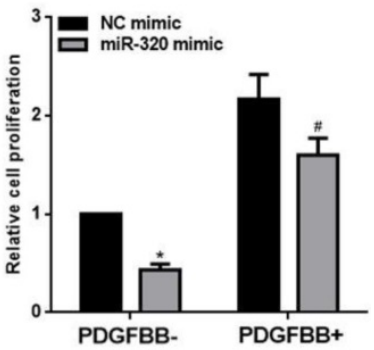

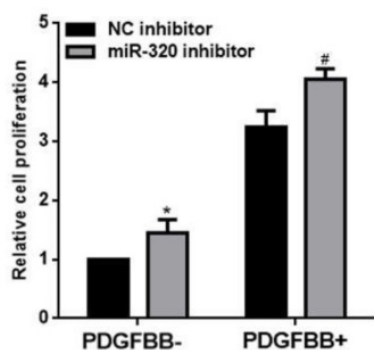

E

$\mathbf{F}$
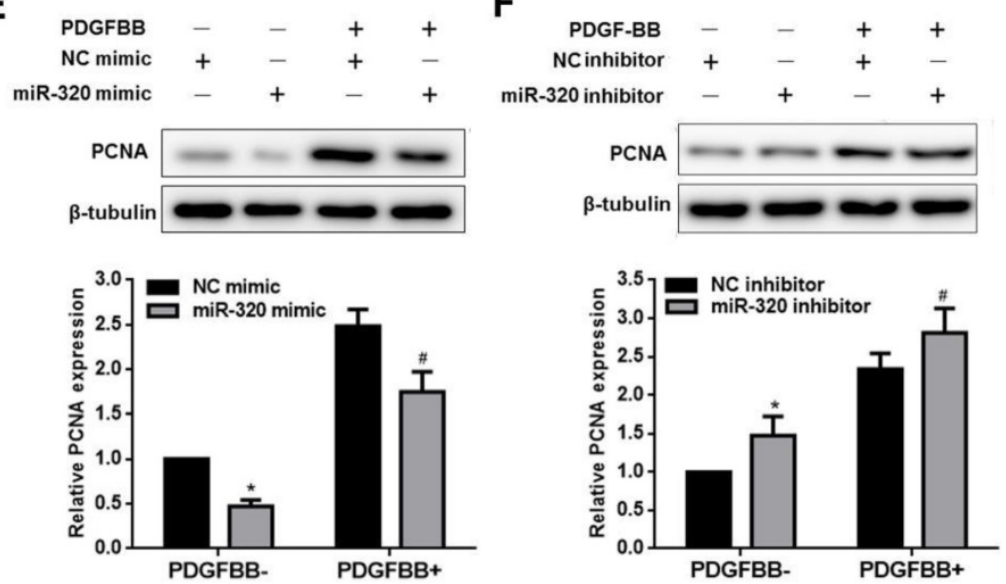

Figure 2. miR-320 inhibits VSMCs proliferation. (A and C) VSMCs were transfected with miR-320 mimic or inhibitor and miR-320 expression was determined by qRT-PCR. (B and D) The EdU incorporation assay detected VSMCs proliferation after transfection with miR-320 mimic or inhibitor with and without PDGF-BB treatment. ( $\mathrm{E}$ and $\mathrm{F}$ ) The expression of PCNA was measured by western blot. $\beta$-tubulin was used as internal control. $\mathrm{n}=3$; **P $<0.01$ vs $\mathrm{NC}$ (negative control), *P $<0.05$ vs NC without PDGF-BB treatment, $\#$ P $<0.05$ vs NC with PDGF-BB treatment.

\section{MiR-320 inhibits VSMCs proliferation and migration}

To identify the effect of miR-320 on VSMCs proliferation and migration, VSMCs were transfected with miR-320 mimic, miR-320 inhibitor or negative control. MiR-320 expression was significantly increased by 92 folds in the mimic group and sharply decreased by $90 \%$ in the inhibitor group (Figure $2 \mathrm{~A}$ and 2C). After transfection with miR-320 mimic, VSMCs proliferation was inhibited with or without PDGF-BB, compared with negative control (Figure 2B). In contrast, we observed that miR-320 inhibitor induced VSMCs proliferation (Figure 2D). Likewise, to further confirm the role of miR-320 in VSMCs proliferation, we detected the expression of a cell proliferation marker, PCNA. PDGF-BB caused a significant increase in PCNA expression as demonstrated by immunoblotting, which was inhibited in miR-320 mimic transfected cells, while miR-320 inhibitor increased PCNA expression (Figure $2 \mathrm{E}$ and $2 \mathrm{~F}$ ).

In addition, we used the wounding-healing assay to measure the effect of miR-320 on VSMCs migration. The overexpression of miR-320 inhibited VSMCs migration with or without PDGF-BB stimulation (Figure 3A). However, VSMCs transfected with miR-320 inhibitor promoted migration (Figure 3B). Together, our results showed that miR-320 is an inhibitor of VSMCs proliferation and migration both basal and PDGF-stimulated conditions. 

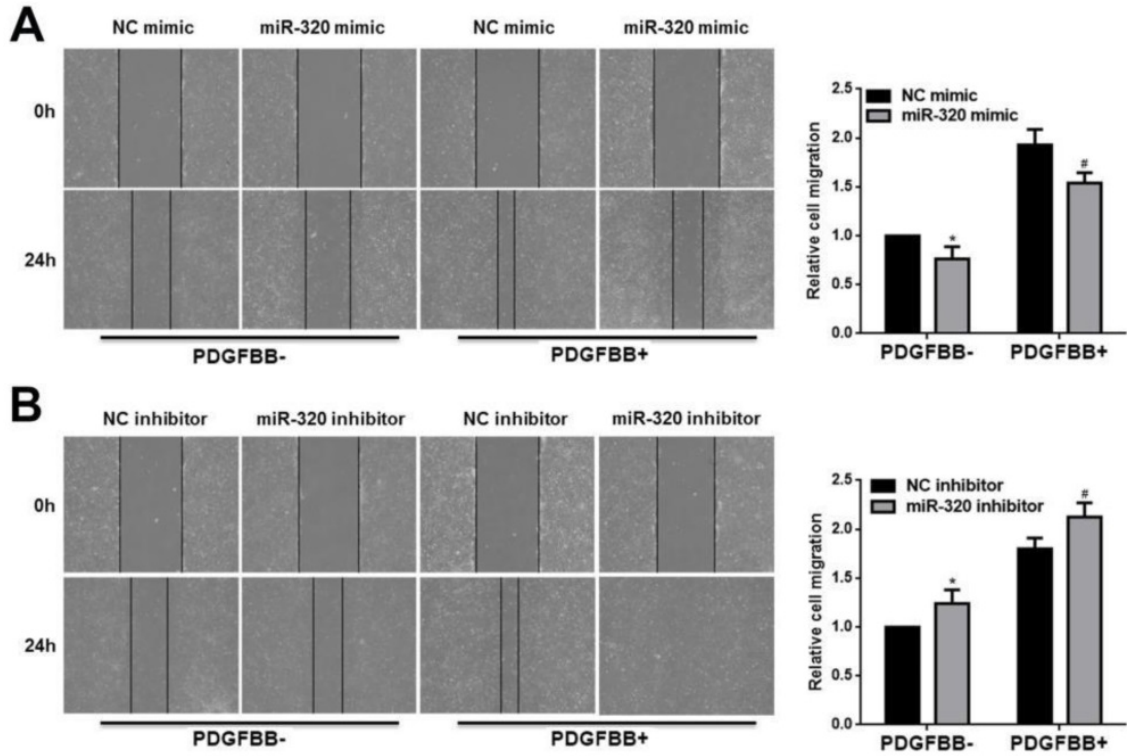

Figure 3. miR-320 inhibits VSMCs migration. $48 \mathrm{~h}$ after transfection with miR-320 mimic or inhibitor, VSMCs were starved for $24 \mathrm{~h}$ and cell migration was evaluated after PDGF-BB treatment for 24h by the wound-healing assay. The images of migrated cells and their respective quantifications were shown (A and B). $n=3$; $* \mathrm{P}<0.05$ vs NC (negative control) without PDGF-BB treatment, \#P $<0.05$ vs NC with PDGF-BB treatment.

A
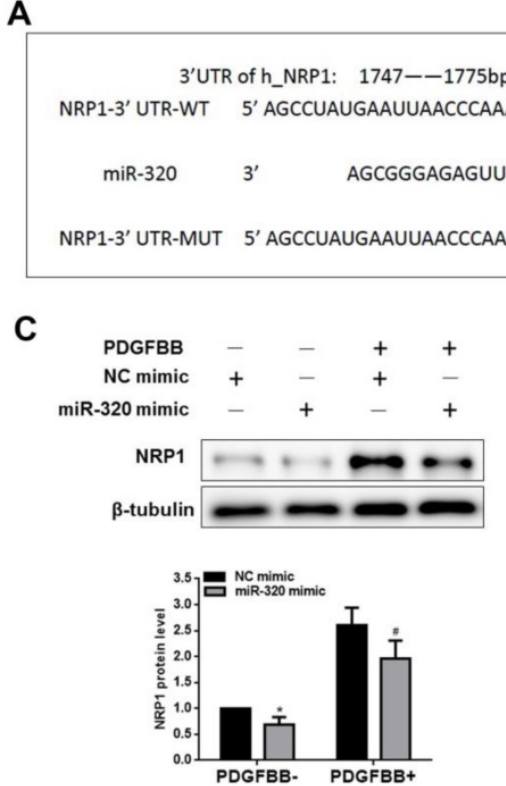

E
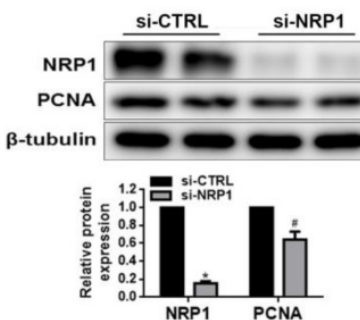

B

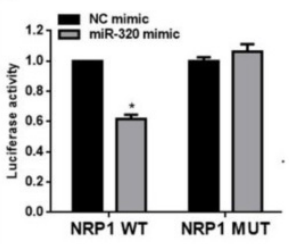

D
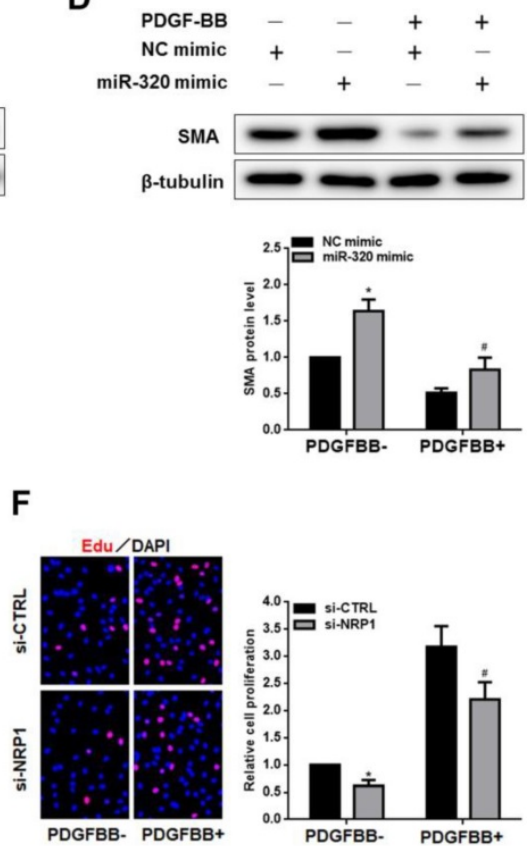

Figure 4. NRPI is a direct target of miR-320 in VSMCs. (A) Diagram of the miR-320 putative binding site in human NRPI 3 '-UTR and alignment of NRP1 wild-type and mutated NRPI 3' UTR binding site of miR-320. The six mutated nucleotides were underlined. (B) miR-320 mimic or NC mimic was cotransfected with NRPI WT or NRP1 Mut construct in HEK293T cells. 48h after transfection, luciferase activity was measured. $n=3$; *P $<0.05$. (C and D) VSMCs were transfected with miR-320 or NC mimic. The protein level of NRPI and SMA was measured by western blot. $n=3$; *P $<0.05$ vs NC (negative control) without PDGF-BB treatment, \#P $<0.05$ vs NC with PDGF-BB treatment. (E) NRP1 and PCNA expression was measured by western blot in VSMCs transfected with si-NRP1. $\mathrm{n}=3$; $* \mathrm{P}<0.05$ vs si-CTRL. (F) The EdU assay detected VSMCs proliferation after transfection with si-NRPI or si-CTRL. $n=3$; *P $<0.05$ vs si-CTRL without PDGF-BB treatment, \#P $<0.05$ vs si-CTRL with PDGF-BB treatment. 


\section{NRPI is a direct target of miR-320}

To search the target of miR-320, we demonstrated NRP1 as a potential target gene by previous studies and TargetScan software. The overexpression of miR-320 significantly suppressed the activity of luciferase with WT-3'-UTR of NRP1. In contrast, the mutation of miR-320-binding sites in the 3'-UTR of NRP1 resulted in the restoration of luciferase activity (Figure 4A and 4B). In addition, the expression of NRP1 and SMA was measured by western blot in VSMCs transfected with either miR-320 mimic or NC mimic. As shown in Figure 4C and $4 \mathrm{D}$, the overexpression of miR-320 sharply repressed NRP1 expression with or without PDGF treatment but enhanced the expression of SMA. These results indicated that NRP1 was a direct target of miR-320 in VSMCs. To further explore the function of NRP1 in VSMCs proliferation, we performed the effect of NRP1 knockdown on VSMCs function. VSMCs transfected with si-NRP1 significantly reduced the expression of NRP1 and PCNA (Figure
4E). In addition, NRP1 knockdown resulted in an obvious decrease in VSMCs proliferation under basal and PDGF-stimulated conditions (Figure 4F).

\section{Identification of miR-320 as a phenotypic marker and NRPI expression in VSMCs of the vascular wall}

To test whether miR-320 is a phenotypic marker in the vascular wall, we established mouse carotid artery ligation model. The left common carotid arteries of mice were collected at 7, 14, 28 days after ligation. The right common carotid arteries without ligation were used as controls. The ligation resulted in time-dependent neointimal hyperplasia in mice carotid arteries (Figure 5A). To examine the distribution of NRP1 expression in the artery, we applied co-immunofluorescence with both NRP1 and SMA at 14 days after ligation. The results demonstrated that NRP1 was mainly distributed in VSMCs of the media of the vascular wall (Figure 5B).

A
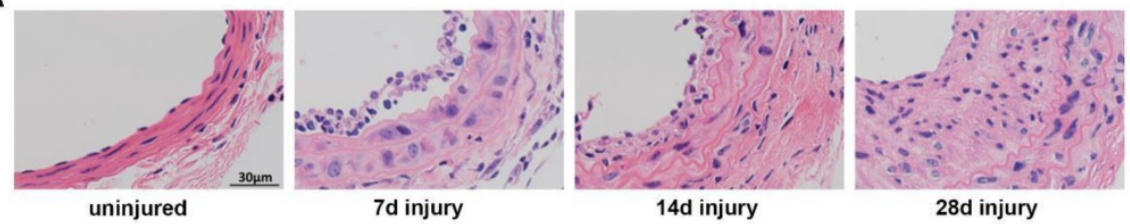

B
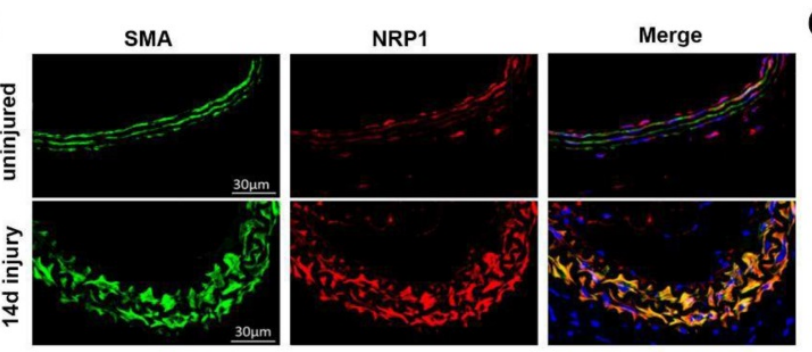

C

D

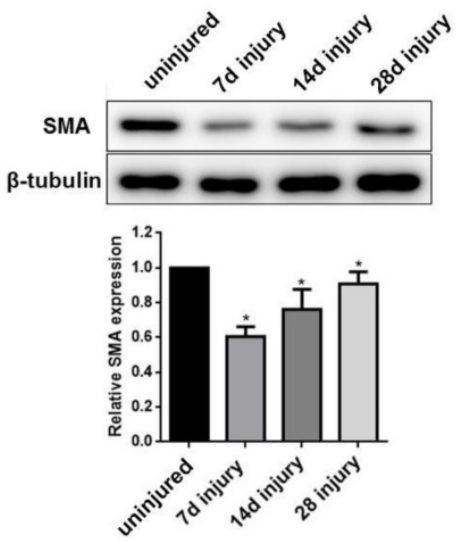

E

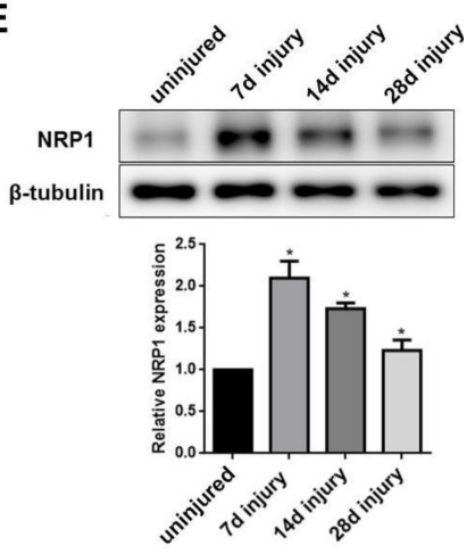

Figure 5. Identification of miR-320 as a phenotypic marker and NRPI expression in VSMCs of the vascular wall. (A) Representative hematoxylin and eosin staining in uninjured and injured mice carotid arteries at 7, 14, and 28 days after ligation. (B) Immunofluorescence with SMA (green), NRPI (red) and merged images in unligated and ligated mice carotid arteries. The Blue was the nucleus stained by DAPI. (C) miR-320 expression in uninjured and injured mice carotid arteries was determined by qRT-PCR. ( $D$ and $E$ ) Representative Western Blot and densitometric analysis of SMA and NRP1 in the vascular wall. $n=5$; $* P<0.05$ vs uninjured group. 


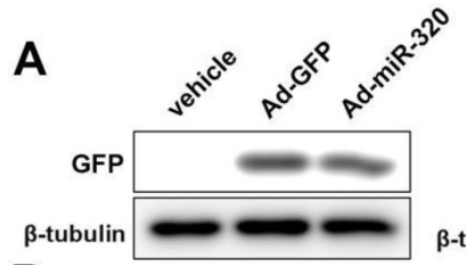

B

E

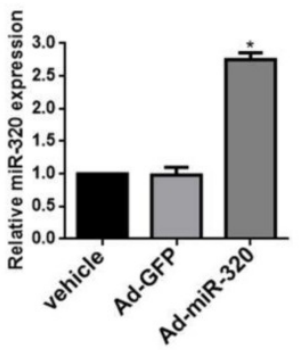

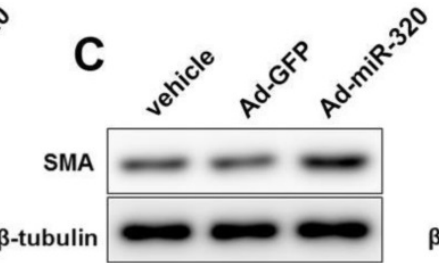

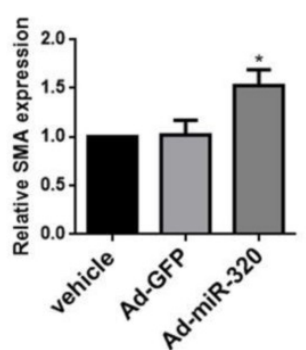

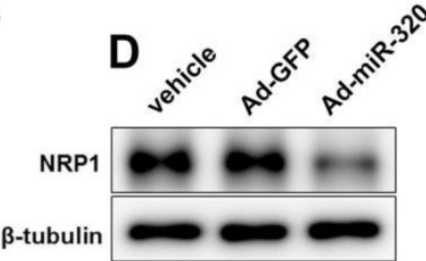

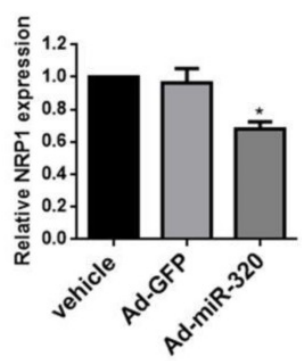

F

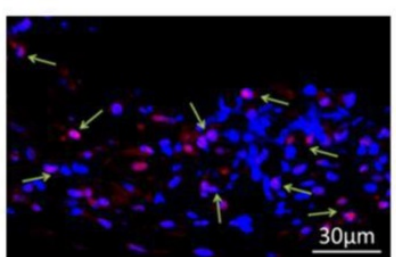

Ad-GFP

G

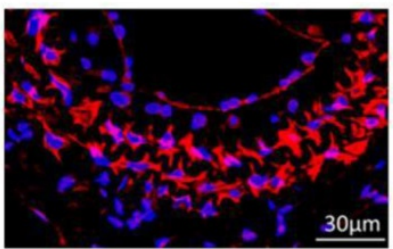

Ad-GFP

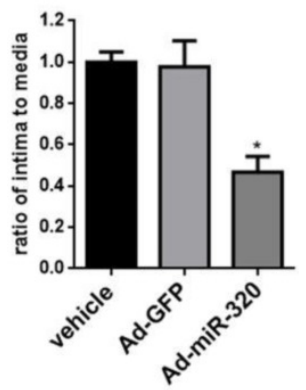

Ad-miR-320

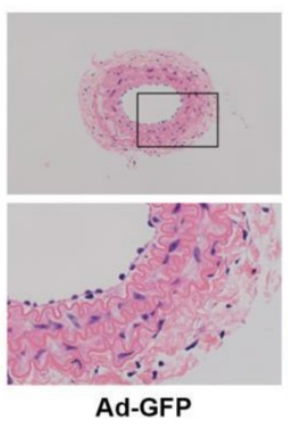

Ad-GFP

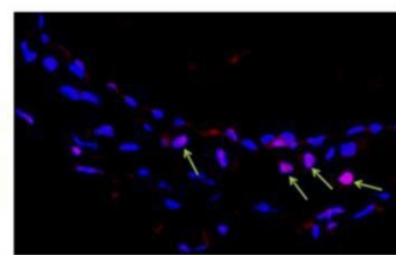

Ad-miR-320
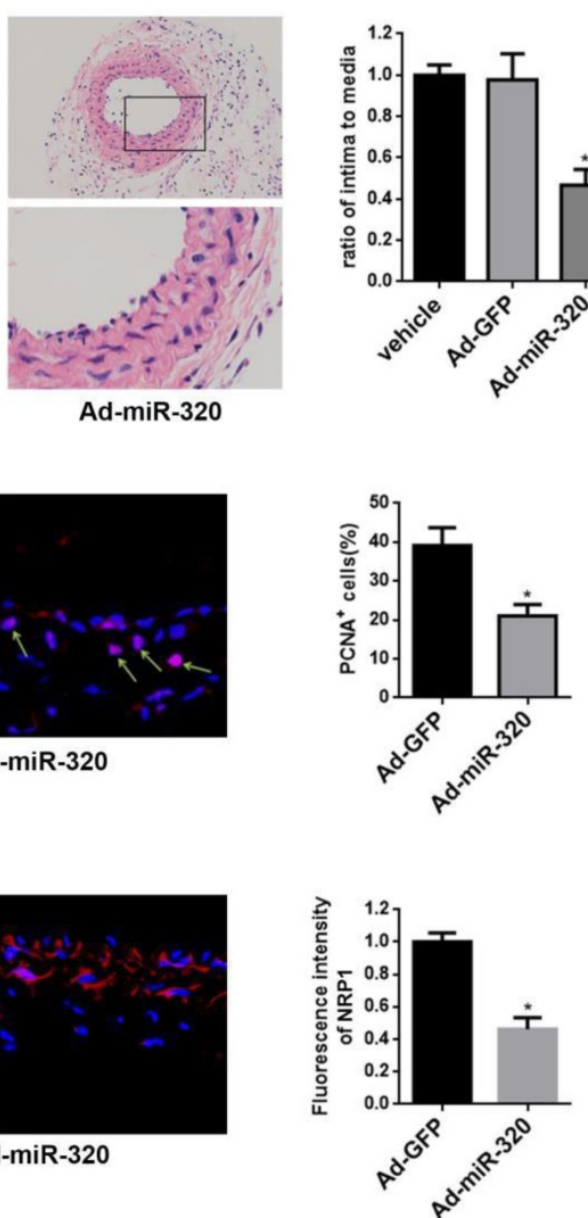

Figure 6. miR-320 inhibits neointimal formation in ligated mice carotid arteries. (A) Representative western blot of GFP in arteries after transfection with Ad-miR-320 or Ad-GFP. (B) The relative expression of miR-320 in the arteries treated with Ad-miR-320, Ad-GFP or vehicle at 14 days after ligation. (C and D) The effect of miR-320 on SMA and NRPI expression in the ligated arteries treated with Ad-miR-320, Ad-GFP or vehicle, and their quantifications were determined by western blot. (E) Representative hematoxylin and eosin staining in mice carotid arteries treated with Ad-miR-320, Ad-GFP or vehicle at 14 days after ligation and intima/media ratio were quantified. (F) Representative Immunofluorescence staining of PCNA (red) and DAPI (blue) in injured carotid arteries treated with Ad-miR-320 or Ad-GFP. The proportion of PCNA-positive cells was calculated. (G) Representative Immunofluorescence staining of NRPI (red) and DAPI (blue) in injured carotid arteries treated with Ad-miR-320 or Ad-GFP. The corresponding fluorescence intensity of NRPI was measured. $n=5$; *P<0.05 vs Ad-GFP group.

In turn, compared with the uninjured group, miR-320 and SMA expression in ligated artery were significantly downregulated as determined by qRT-PCR or western blot, respectively (Figure 5C and 5D). In contrast, NRP1 expression was significantly upregulated after ligation (Figure 5E). These findings suggested that miR-320 played important role in the phenotypic switch and NRP1 was located in media of the vascular wall. 


\section{MiR-320 inhibits neointimal formation in ligated mice carotid arteries}

To restore the miR-320 expression in the injured vessel, carotid arteries were treated with Ad-miR-320 $\left(10^{10} \mathrm{pfu} / \mathrm{mL}\right)$, Ad-GFP or vehicle. At 14 days after transfection, the expression of GFP was detected in Ad-GFP group and Ad-miR-320 group and miR-320 expression in Ad-miR-320 group was significantly increased compared with either vehicle or Ad-GFP group by qRT-PCR (Figure 6A and 6B). Next, the restoration of miR-320 sharply upregulated the SMA expression compared with arteries treated with vehicle or Ad-GFP determined by western blot (Figure 6C). However, as shown in Figure 6D, the expression of NRP1 in mice carotid arteries was down-regulated via Ad-miR-320 treatment.

To confirm the role of miR-320 in neointimal growth, we examined carotid intima-media thickness. Neointimal formation was markedly attenuated in ligated mice carotid arteries transfection with Ad-miR-320. Representative hematoxylin and eosin-stained images of mice carotid arteries from the vehicle, Ad-GFP, and Ad-miR-320 groups were shown (Figure 6E). Immunofluorescence staining of PCNA and NRP1 was applied in Ad-miR-320 or Ad-GFP carotid arteries at 14 days after the injury. The results revealed that the number of PCNA-positive cells decreased in Ad-miR-320 arteries compared with Ad-GFP arteries (Figure 6F). Furthermore, the expression of NRP1 also declined in the arteries treated with Ad-miR-320 (Figure 6G). Collectively, these results suggest that miR-320 suppresses neointima formation by inhibiting NRP1-mediated VSMCs proliferation.

\section{Discussion}

In the current study, we identified miR-320 as a novel modulator involved in VSMCs proliferation and migration and neointimal formation. We demonstrated that miR-320 was dramatically down-regulated in PDGF-treated VSMCs. Downregulation of miR-320 promoted the proliferation and migration of VSMCs, while the restoration of decreased miR-320 could inhibit VSMCs proliferation and migration in vitro. Likewise, miR-320 was also significantly decreased in mice carotid arteries after ligated injury. The gain-of-function studies indicated that miR-320 attenuated neointimal formation in vivo. Moreover, miR-320 inhibited VSMCs proliferation and migration in vitro and vivo by targeting NRP1.

Increasing studies indicate that miR-320 is associated with cell differentiation, proliferation, migration, and apoptosis [20, 24, 25]. It has been reported that miR-320 is a tumor suppression miRNA and inhibits cancer progression and metastasis [26]. Nevertheless, the underlying mechanism of miR-320 in VSMCs biology still remains unclear. In our study, we identified that miR-320 was significantly associated with PDGF-stimulated VSMCs proliferation and migration. The inhibition of miR-320 expression promoted VSMCs proliferation and migration, while miR-320 overexpression performed the opposite effect. Moreover, we revealed that the expression changes of miR-320 were consistent with SMA, a differentiation marker gene of VSMCs. These results demonstrated that miR-320 modulated proliferation and phenotypic switch for VSMCs in vitro.

MiRNAs regulate the biological function of cells by interacting with multiple mRNA targets. Firstly, based on the inhibition of miR-320 on VSMCs proliferation, we assumed that target genes could be positively correlated with cell growth. NRP1 is a kind of membrane-binding co-receptor of tyrosine kinase receptor and plays an important role in angiogenesis and tumor invasion and metastasis. In VSMCs, NRP1 interacted with PDGF- $a$ and was involved in PDGF-stimulated VSMCs migration by $\mathrm{p} 130^{\mathrm{cas}}[27,28]$. In addition, PDGF physically interacted with NRP1, while it induced the migration of VSMCs through NRP1 in breast cancer [29]. In mesenchymal stem cells with the function of differentiating into VSMCs, NRP1 promoted PDGF-induced migration and proliferation [30]. In our study, TargetScan predicted the target gene of miR-320, which identified that NRP1 had binding sites for miR-320. Effect of miR-320 on NRP1 was further confirmed in HEK293T by Luciferase assay. The expression of NRP1 performed a pronounced upregulation in VSMCs treated with PDGF, while miR-320 overexpression negatively regulated the expression of NRP1. In addition, we also found that knockdown of NRP1 suppressed VSMCS proliferation, which was consistent with the results that miR-320 inhibited the proliferation of VSMCs.

Multiple miRNAs were found to be involved in the neointimal formation, such as miR-21, miR-145 and miR-221/222, which affected cell apoptosis, differentiation and proliferation [15, 16, 23]. After balloon injury, the expression of miR-221/222 and miR-21 was upregulated. In contrast, miR-145 has the highest abundance in VSMCs of the vascular wall and significantly decreased after vascular injury. The restoration of miR-145 inhibits neointimal formation, which indicated that miR-145 had a potential therapeutic effect [15]. miR-320 is widely expressed in mammals, such as humans, rats and mice and the binding sequences of miR-320 in NRP1 $3^{\prime}$-UTR is highly conserved among different species, which has prompted us to verify the function of miR-320 by 
mouse carotid artery ligation model. We found that NRP1 was expressed in VSMCs of the vascular wall and the protein level was significantly upregulated in injured artery. To determine the distribution of NRP1 in vascular wall, immunofluorescence staining confirmed that NRP1 was located in VSMCs of the carotid arteries. Moreover, the restoration of decreased miR-320 by Ad-miR-320 significantly attenuated the neointimal formation, accompanied with the down-regulated expression of NRP1 and PCNA. Likewise, to investigate the effect of miR-320 on the phenotype of VSMCs, we found that the expression of SMA has been down-regulated after the injury, while overexpressing miR-320 partially restored the expression of SMA. Our results suggest that the miR-320 regulated the proliferation and migration of VSMCs by targeting the NRP1.

In summary, we demonstrated that the expression of miR-320 rapidly decreased in VSMC by PDGF or vascular injury. The downregulation of miR-320 increases the expression of its target gene NRP1, which has promoted VSMCs proliferation and resulted in neointimal formation. The regulation of NRP1 expression by miR-320 has important significance in the diagnosis and treatment of cardiovascular diseases, such as in-stent restenosis and atherosclerosis.

\section{Acknowledgments}

This work was supported by the National Natural Science Foundation of China (81270193) and the Natural Science Foundation of Shanghai (18ZR1429700).

\section{Competing Interests}

The authors have declared that no competing interest exists.

\section{References}

1. Marx SO, Totary-Jain H, Marks AR. Vascular smooth muscle cell proliferation in restenosis. Circulation Cardiovascular interventions. 2011; 4: 104-11.

2. McDonald RA, Halliday CA, Miller AM, Diver LA, Dakin RS, Montgomery I, et al. Reducing In-Stent Restenosis: Therapeutic Manipulation of miRNA in Vascular Remodeling and Inflammation. Journal of the American College of Cardiology. 2015; 65: 2314-27.

3. Raines EW. PDGF and cardiovascular disease. Cytokine \& growth factor reviews. 2004; 15: 237-54

4. Guo H, Ingolia NT, Weissman JS, Bartel DP. Mammalian microRNAs predominantly act to decrease target mRNA levels. Nature. 2010; 466: 835-40.

5. Huntzinger E, Izaurralde E. Gene silencing by microRNAs: contributions of translational repression and mRNA decay. Nature reviews Genetics. 2011; 12: 99-110.

6. Ambros V. The functions of animal microRNAs. Nature. 2004; 431: 350-5.

7. Zhao Y, Samal E, Srivastava D. Serum response factor regulates a muscle-specific microRNA that targets Hand2 during cardiogenesis. Nature. 2005; 436: 214-20.

8. Zhang C. MicroRNomics: a newly emerging approach for disease biology. Physiological genomics. 2008; 33: 139-47.

9. Xu P, Guo M, Hay BA. MicroRNAs and the regulation of cell death. Trends in genetics : TIG. 2004; 20: 617-24

10. Lee CT, Risom T, Strauss WM. MicroRNAs in mammalian development. Birth defects research Part C, Embryo today : reviews. 2006; 78: 129-39.
11. Zhang C. MicroRNAs: role in cardiovascular biology and disease. Clinical science (London, England : 1979). 2008; 114: 699-706.

12. Xie C, Huang H, Sun X, Guo Y, Hamblin M, Ritchie RP, et al. MicroRNA-1 regulates smooth muscle cell differentiation by repressing Kruppel-like factor 4. Stem cells and development. 2011; 20: 205-10.

13. Wang M, Li W, Chang GQ, Ye CS, Ou JS, Li XX, et al. MicroRNA-21 regulates vascular smooth muscle cell function via targeting tropomyosin 1 in arteriosclerosis obliterans of lower extremities. Arteriosclerosis, thrombosis, and vascular biology. 2011; 31: 2044-53.

14. Chan MC, Hilyard AC, Wu C, Davis BN, Hill NS, Lal A, et al. Molecular basis for antagonism between PDGF and the TGFbeta family of signalling pathways by control of miR-24 expression. The EMBO journal. 2010; 29: 559-73.

15. Cheng Y, Liu X, Yang J, Lin Y, Xu DZ, Lu Q, et al. MicroRNA-145, a novel smooth muscle cell phenotypic marker and modulator, controls vascular neointimal lesion formation. Circulation research. 2009; 105: 158-66.

16. Liu X, Cheng Y, Zhang S, Lin Y, Yang J, Zhang C. A necessary role of miR-221 and miR-222 in vascular smooth muscle cell proliferation and neointimal hyperplasia. Circulation research. 2009; 104: 476-87.

17. Li P, Zhu N, Yi B, Wang N, Chen M, You X, et al. MicroRNA-663 regulates human vascular smooth muscle cell phenotypic switch and vascular neointimal formation. Circulation research. 2013; 113: 1117-27.

18. Wu YY, Chen YL, Jao YC, Hsieh IS, Chang KC, Hong TM. miR-320 regulates tumor angiogenesis driven by vascular endothelial cells in oral cancer by silencing neuropilin 1. Angiogenesis. 2014; 17: 247-60.

19. Zhu H, Jiang X, Zhou X, Dong X, Xie K, Yang C, et al. Neuropilin-1 regulated by miR-320 contributes to the growth and metastasis of cholangiocarcinoma cells. Liver international: official journal of the International Association for the Study of the Liver. 2018; 38: 125-35.

20. Hsieh IS, Chang KC, Tsai YT, Ke JY, Lu PJ, Lee KH, et al. MicroRNA-320 suppresses the stem cell-like characteristics of prostate cancer cells by downregulating the Wnt/beta-catenin signaling pathway. Carcinogenesis. 2013; 34: 530-8.

21. Vishnubalaji R, Hamam R, Yue S, Al-Obeed O, Kassem M, Liu FF, et al. MicroRNA-320 suppresses colorectal cancer by targeting SOX4, FOXM1, and FOXO1. Oncotarget. 2016; 7: 35789-802.

22. Satoh K, Matoba T, Suzuki J, O'Dell MR, Nigro P, Cui Z, et al. Cyclophilin A mediates vascular remodeling by promoting inflammation and vascular smooth muscle cell proliferation. Circulation. 2008; 117: 3088-98.

23. Ji R, Cheng Y, Yue J, Yang J, Liu X, Chen H, et al. MicroRNA expression signature and antisense-mediated depletion reveal an essential role of MicroRNA in vascular neointimal lesion formation. Circulation research. 2007; 100: 1579-88.

24. Wan LY, Deng J, Xiang XJ, Zhang L, Yu F, Chen J, et al. miR-320 enhances the sensitivity of human colon cancer cells to chemoradiotherapy in vitro by targeting FOXM1. Biochemical and biophysical research communications. 2015; 457: 125-32.

25. Lei T, Zhu Y, Jiang C, Wang Y, Fu J, Fan Z, et al. MicroRNA-320 was downregulated in non-small cell lung cancer and inhibited cell proliferation, migration and invasion by targeting fatty acid synthase. Molecular medicine reports. 2016; 14: 1255-62.

26. Bronisz A, Godlewski J, Wallace JA, Merchant AS, Nowicki MO, Mathsyaraja $\mathrm{H}$, et al. Reprogramming of the tumour microenvironment by stromal PTEN-regulated miR-320. Nature cell biology. 2011; 14: 159-67.

27. Pellet-Many C, Frankel P, Evans IM, Herzog B, Junemann-Ramirez M, Zachary IC Neuropilin-1 mediates PDGF stimulation of vascular smooth muscle cell migration and signalling via p130Cas. The Biochemical journal. 2011; 435: 609-18.

28. Kofler N, Simons M. The expanding role of neuropilin: regulation of transforming growth factor-beta and platelet-derived growth factor signaling in the vasculature. Current opinion in hematology. 2016; 23: 260-7.

29. Banerjee S, Sengupta K, Dhar K, Mehta S, D'Amore PA, Dhar G, et al. Breast cancer cells secreted platelet-derived growth factor-induced motility of vascular smooth muscle cells is mediated through neuropilin-1. Molecular carcinogenesis. 2006; 45: 871-80.

30. Ball SG, Bayley C, Shuttleworth CA, Kielty CM. Neuropilin-1 regulates platelet-derived growth factor receptor signalling in mesenchymal stem cells. The Biochemical journal. 2010; 427: 29-40. 